Research Article

\title{
Track Irregularity Assessment in High-Speed Rail by Incorporating Carriage-Body Acceleration with Transfer Function
}

\author{
Liubin Niu $\mathbb{D}^{1},{ }^{1}$ Jinzhao Liu $\mathbb{D}^{1},{ }^{1}$ and Yunlai Zhou $\mathbb{D}^{2}$ \\ ${ }^{1}$ Infrastructure Inspection Institute of China Railway Sciences Institute Group Co. Ltd., 100081 Beijing, China \\ ${ }^{2}$ State Key Laboratory for Strength and Vibration of Mechanical Structures, School of Aerospace Engineering, \\ Xi'an Jiaotong University, Xi'an 710049, China \\ Correspondence should be addressed to Jinzhao Liu; liujinzhao_2010@sina.com
}

Received 5 October 2019; Revised 7 March 2020; Accepted 20 March 2020; Published 27 June 2020

Academic Editor: Lukasz Pieczonka

Copyright ( $\odot 2020$ Liubin Niu et al. This is an open access article distributed under the Creative Commons Attribution License, which permits unrestricted use, distribution, and reproduction in any medium, provided the original work is properly cited.

\begin{abstract}
The determination of the precise track irregularity with unfavorable wavelength, which shall induce vehicle's violent vibration in terms of the vehicle's speeds, still challenges the researchers. This study proposes a feasible study of assessing the track irregularity by using the transfer function and the measured carriage-body acceleration by combining the ARX model with state space model. The ARX model and state space model are constructed using system identification to obtain the transfer relation between the track irregularity and the carriage-body acceleration, respectively. The model's parameters are estimated by the measured data from the high-speed China Railway Comprehensive Inspection Train (CRCIT). The correlation value between the predicted and measured carriage-body acceleration shows that both models can effectively represent the transfer characteristics between the track irregularity and the carriage-body acceleration. Furthermore, the models can help assess the proportion of the vibration caused by track irregularity with the specific wavelengths and determine the track irregularity with unfavorable wavelength.
\end{abstract}

\section{Introduction}

In railway systems, vibration has been a severe problem, which has several forms: track irregularity, wheel defect like polygonization, vortex-induced vibration (VIV), velocity-induced vibration, and so on. VIV exists mostly and commonly in the high-speed railway (HSR) system. In all HSR systems, the track irregularity-induced vibration certainly serves as the main source and has attracted lots of attention in both engineering and scientific committees $[1,2]$.

In terms of track irregularity, normally referring to the deviation of the geometry, size and location of the track relative to its normal pattern depicted in Figure 1, which can be divided into static irregularity and dynamic irregularity according to whether there is trainload or not. It generates certain level of vibration in the passing carriages. For characterizing consideration, track irregularity can be illustrated with profile, alignment, cross level, gauge, and twist in the eye of vertical, lateral, and horizontal directions. On the other hand, it can also be explained with amplitude and wavelength from the perspective of wave propagation theory including short-wave, medium-wave, and long-wave irregularities. Such track irregularity shall probably has certain influence on the stability, comfortability and safety as well for the passing carriages especially in HSR systems.

Regarding the track, since it suffers from the repeated high-speed passing trainload, poor quality track shall bring severe vibration in the passing carriages and ambient noise radiation and accelerate the degradation of track and its related infrastructures, leading to the aggravation in damage of the track in a vicious circle. However, good quality track shall both ensure the operating safety and guarantee the comfortability and stability for passengers. Meanwhile, good quality track shall also highly extend the periodic maintenance interval, leading to the cost reduction in a virtuous cycle.

As illustrated above, the track irregularity has profound effect in the HSR system especially to the track. In order to characterize the track irregularity, a lot of approaches and techniques have been raised, like laser scanning, video 

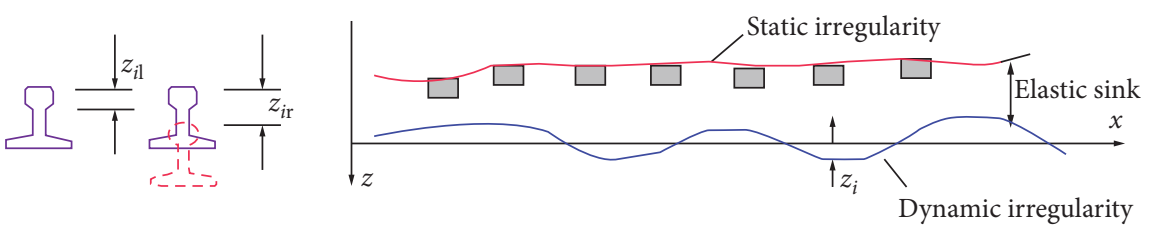

(a)
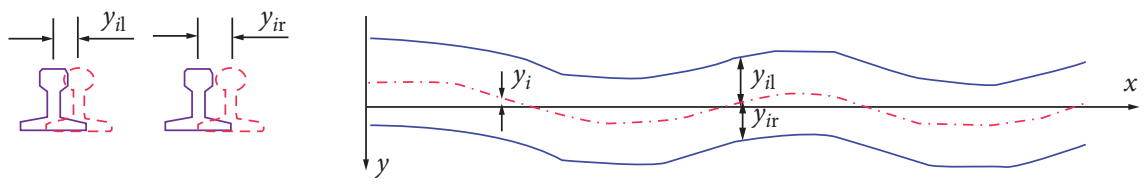

(b)
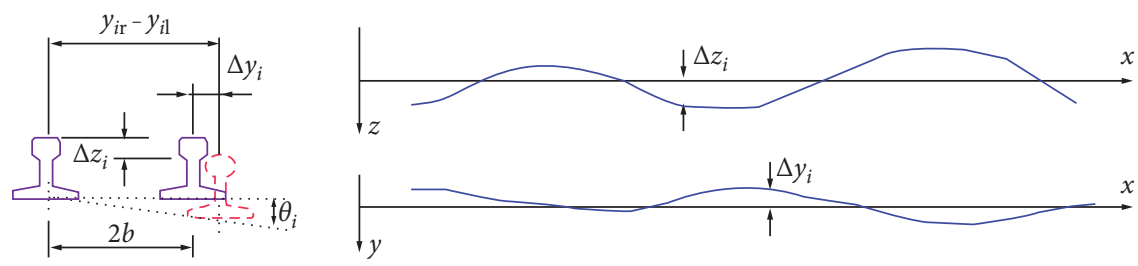

(c)

Figure 1: Illustration of track irregularities. (a) Vertical profile. (b) Alignment. (c) Cross level and gauge.

capture, vibration acceleration, and so on. These techniques can be categorized into direct techniques and indirect techniques. Direct techniques aim to exactly measure the track irregularity with the size, location, and so on, such as laser scanning and video capture. Indirect techniques try to use indirect measurement like accelerations at the axial box or the carriage body to reflect the status of the irregularity in the track. Related approaches to these direct and indirect techniques include image processing, data processing, and so on. Among all these techniques, the acceleration measurement at the carriage body comes as the most economic but effective manner in detecting the track irregularity with the unfavorable wavelength. Certainly, the acceleration at the carriage body includes the complex components induced by the track irregularity and also the transfer features of the carriages.

When the track is under relative good condition, the approximate linear transfer relation between the track irregularity profile and the carriage-body vertical acceleration can be captured and analyzed. Compared with the vertical transmission, the impact on the lateral acceleration of the carriage body from the alignment varies with the frequency, and there exists an increasingly nonlinear transfer relation from alignment, cant, and crosslevel to the carriage-body lateral acceleration, especially with the track degradation after long-term use. Such study on the relation from track irregularity to the dynamic response of the carriages helps the understanding of how the track defects affect the responses of the carriages and provide maintenance suggestions for the engineering fields. Previous studies have been highly conducted on such track irregularity and its consequences on the vehicle dynamic responses with kinds of approaches like transfer function [3], Kalman transform [4], short-time Fourier transform [5], and wavelet analysis [6]. The transfer characteristics between the track irregularity and the vehicle vibration are studied by means of vehicletrack coupling dynamic theory and frequency analysis method [7-9]. The transfer characteristics between the track irregularity and the vehicle vibration are studied by backpropagation (BP) and linear parametric model [10, 11]. Since comfortability in operation can be calculated from the carriage-body acceleration, the relation between the track irregularities and the comfortability in operation is studied $[5,12,13]$. Most of the above studies used signal processing methods such as coherent analysis and Kalman filter or vehicle-track dynamic theory to study the relation between the track irregularity and the carriage-body acceleration through spectrum analysis, focusing on determining the quantitative distribution of single track irregularity wavelength which can cause severe vehicle vibration, but they cannot find out explicit transfer characteristics.

To investigate the transfer relation between the track irregularity and the carriage-body acceleration, this study constructs the autoregressive with exogenous input (ARX) model and multi-input-single-output (MISO) state space model to obtain the relation between the track irregularity and the carriage-body vertical and lateral accelerations, respectively, based on system identification theory and verifies the models by the measured data from the high-speed China Railway Comprehensive Inspection Train (CRCIT), as shown in Figure 2, which can detect the track irregularity with the maximum detection wavelength of $150 \mathrm{~m}$.

In this study, the track irregularity profile was defined as the mean value of the left and right side measured track irregularity profile, which contains wavelength ranges from 1.5 to $150 \mathrm{~m}$. The alignment was defined similarly as the mean value of the left and right side measured alignment, which contains wavelength ranging from 1.5 to $150 \mathrm{~m}$. The short-wave track irregularity with wavelength less than $1.5 \mathrm{~m}$ 


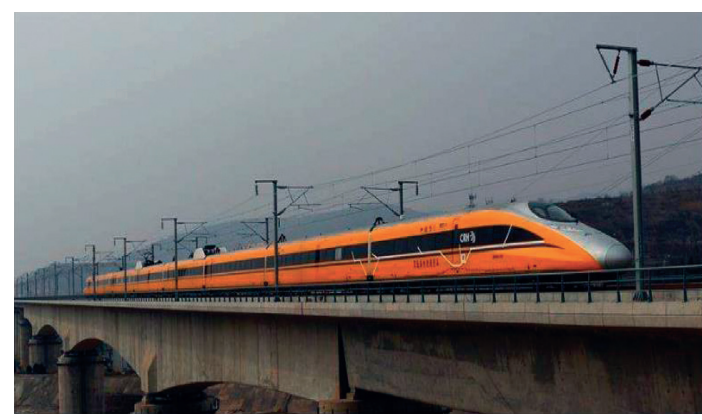

Figure 2: The Chinese high-speed railway comprehensive inspection train (CRCIT).

has little effect on the carriage-body vertical and lateral acceleration, and the vehicle's vibration caused by the short-wave track irregularity is ignored. In order to study the relation between the track irregularity and the carriage-body acceleration in wavelength ranging from 1.5 to $150 \mathrm{~m}$, bandpass filtering is applied to the measured data in advance. The measured data are selected from some HSR with the running speed of $200 \mathrm{~km} / \mathrm{h}$ and the sampling interval of $0.25 \mathrm{~m}$.

\section{Transfer Model Derivation}

Upon system identification theory, the transfer model between the track irregularity (the input) and the carriagebody acceleration (the output) is constructed. Firstly, to obtain the main factor and simplify the input of the model, coherent analysis is applied to find the coherence characteristics between track irregularity and the carriage-body acceleration in different spatial frequency bands, and the suitable inputs are selected; secondly, the model order and corresponding model parameters are determined based on the measured data from the CRCIT. Finally, the model's accuracy is verified by contrasting the measured carriagebody acceleration and the predicted data from the model transfer function.

2.1. Coherence Function. Before building the model, the track irregularity that has strong coherence with the carriage-body acceleration is defined as the input. The coherence function can characterize the coherence characteristics between the input and output of the model, and it can illustrate the degree of dependence among the track irregularity $u$ and the carriage-body acceleration $y$ in different spatial frequency bands.

The coherence function $C_{u y}$ is defined as

$$
C_{u y}(\omega)=\frac{\left|C_{u y}(\omega)\right|^{2}}{C_{y y}(\omega) C_{u u}(\omega)},
$$

where $\omega$ is the spatial frequency, $G_{u y}(\omega)$ is the cross-power spectrum density (PSD) of the track irregularity $u$ and the carriage-body acceleration $y, G_{u u}(\omega)$ is the auto PSD of track irregularity $u$, and $G_{y y}(\omega)$ is the auto PSD of the carriagebody acceleration. For more details on autopower spectrum and cross-power spectrum analysis method, one can refer to
[14]. In this study, the PSDs of the track irregularity and the carriage-body acceleration are computed in units of power per radians per sample using the averaged modified periodogram spectral estimation method. The estimated data are segmented into eight sections on average, each with $50 \%$ overlap, and each segment is windowed with a hamming window at the same length as the segment. The number of Fourier transform spectrum lines in each segment is 4096.

The coherence function value ranges from 0.0 to 1.0 ; the closer the coherence function value is to 1.0, the stronger the dependency between the inputs and output of the model will be. When the coherence function value is greater than 0.8 , strong coherence relation between two sets of data exists.

2.2. Vertical Transfer Model. A quasilinear relation between the track irregularity profile and the carriage-body vertical acceleration can be modeled by the linear model [15]. The input of the linear model is selected by coherent analysis. When the test noise of the measured data is small, the loworder ARX model has few parameters to be determined and its algorithm is simple. When the noise of the measured data is large, the model order has to be increased accordingly to compensate the noise effect on the identification accuracy. This study uses the ARX model to estimate the transfer relation between the track irregularity profile and the carriage-body vertical acceleration. The model can be described as

$$
\begin{aligned}
& y(t)+a_{1} y(t-1)+\cdots+a_{n a} y(t-n a) \\
& =b_{1} u(t-n k)+\cdots+b_{n b} u(t-n k-n b+1)+v(t)
\end{aligned}
$$

where $y(t)$ is the output value at time $t, y(t-1)$ and $y(t-n a)$ are the output values at time $t-1$ and $t-n a$, respectively, and $v(t)$ is the test noise value at time $t$; the noise is an additional unmeasurable signal; it is assumed that the effect on the output of the noise is insignificant compared to the effect on the input. Similarly, $u(t-n k)$ and $u(t-n k-n b+1)$ are the input value at time $t-n k$ and $t-n k-n b+1$.

The parameter $n k$ is the delay number from input to output of the ARX model; the bigger the number $n k$ is, the greater the delay between the input and the output will be. The ARX model can adopt one-time delay, and the value $n k$ is set to 1. $n a$ and $n b$ are the orders of the model. $a_{1}, a_{2}, \ldots, a_{n a}$ and $b_{1}, b_{2}, \ldots, b_{n b}$ are the model parameters to be identified.

The unit delay operator $q$ is introduced; equation (2) could be simplified as

$$
A(q) y(t)=B(q) u(t)+v(t)
$$

where

$$
\begin{aligned}
& q^{-k} u(t)=u(t-k), \\
& \left\{\begin{array}{l}
A(q)=1+a_{1} q^{-1}+a_{2} q^{-2}+\cdots+a_{n a} q^{-n a}, \\
B(q)=b_{1} q^{-n k}+b_{2} q^{-n k-1}+\cdots+b_{n b} q^{-n k-n b+1}
\end{array}\right.
\end{aligned}
$$

The ARX model between track irregularity profile and the carriage-body vertical acceleration can be regarded as a linear system; equation (3) can be described as 


$$
y(t)=G(q) u(t)+e(t),
$$

where $G(q)$ is the transfer function of the ARX model and its expression is as follows:

$$
y(q)=\frac{A(q)}{B(q)} .
$$

The prediction output can be calculated as

$$
\widehat{y}(t)=\widehat{G}(q) u(t)=\widehat{B}(q) u(t)+[1-\widehat{A}(q)] \widehat{y}(t),
$$

where $u(t)$ is measured profile, mm. $\hat{y}(t)$ is the predicted output from $u(t)$ and the transfer function of the ARX model. $\widehat{G}(q)$ and $\widehat{A}(q), \widehat{B}(q)$ are respective the transfer function and the polynomial parameters of the constructed model.

The key to identifying the transfer relation between the input and the output is to accurately estimate the parameters, $a_{1}, a_{2}, \ldots, a_{n a}, b_{1}, b_{2}, \ldots, b_{n b}$. The least square method algorithm is used in the process of estimating the parameters, which means the appropriate $A(q)$ and $B(q)$ are selected to minimize the model loss function. The loss function is defined as

$$
V=\sum_{t=1}^{N}\{\widehat{y}(t)-y(t)\}^{2}
$$

where $\hat{y}(t)$ is the predicted output from the model and $y(t)$ is the measured output. The number $N$ is the total amount of the measured data.

This study uses the minimum FPE (final prediction error) criterion or Akaike information criterion (AIC) to find the minimum of the loss function and determine the order $n a, n b$ and the parameters $A(q), B(q)$ accordingly. The FPE and AIC guidelines are defined as

$$
\begin{aligned}
& \mathrm{FPE}=V \frac{1+(d / N)}{1-(d / N)}, \\
& \mathrm{AIC}=\log (V)+\frac{2 d}{N},
\end{aligned}
$$

where $d$ is the sum of $n a$ and $n b$.

2.3. Lateral Transfer Model. As to the nonlinear relation between the track irregularities and the carriage-body lateral acceleration, a multivariable state space model with multiple inputs and one output can be used to indicate the relation.

The inputs are also selected by use of the coherence analysis mentioned in Section 2.1 in different spatial frequency bands. By using the state space model $[16,17]$, the relation between the input $u$ and the output $y$ can be described as

$$
\left\{\begin{array}{l}
x(t+1)=A x(t)+B u(t)+v_{1}(t), \\
y(t)=C x(t)+D u(t)+v_{2}(t) .
\end{array}\right.
$$

For the state space model, the input $u(t)$ and output $y(t)$ at time $t$ are related via the state variable $x(t)$ of the model. $v_{1}(t)$ and $v_{2}(t)$ are the model noise signals at time $t . A, B, C$, and $D$ are the matrix parameters to be determined by the subspace algorithm, whose dimensions depend on the model order and the dimension of the input and output. Assuming the model order is $n$, the dimension of the input is $m \times n$ and the dimension of the output is $r \times 1$; matrix $A$ is a $n \times n$ matrix, $B$ is a $n \times m$ matrix, $C$ is a $r \times n$ matrix, and $D$ is a $m \times r$ matrix. The value of matrixes $A, B, C$, and $D$ is also estimated by the measured data from the CRCIT.

After the matrixes are well determined, the transfer function $G(\omega)$ of the state space model can be computed as follows:

$$
G(\omega)=C\left(\omega I_{n}-A\right)^{-1} B+D,
$$

where $\omega$ is the spatial frequency and $I_{n}$ is an $n^{\text {th }}$ order identity matrix.

The output of the state space model can also be predicted from the model transfer function and the measured input. The algorithm is described as

$$
\widehat{y}(k)=\widehat{G}(\omega) u(t),
$$

where $\hat{y}(k)$ is the predicted output; $\widehat{G}(\omega)$ is the transfer function of the state space model computed by equation (12); and $u(t)$ is the measured input.

Selecting a reasonable model order will help to optimize the number of the model parameters and to improve the system identification accuracy. A reasonable order and corresponding model parameters of the state space model are obtained by the loss function and the FPE criterion in equations (8) and (9) as well.

This study adopts a one-step forward prediction method when using the transfer function to predict the output. The predication value is calculated from the $n^{\text {th }}$ order state space model at the time $k ; \hat{y}(k)$ is set to 0 in advance when $k$ is less than the order of the state space model. $\hat{y}(1)=\hat{y}(2)=\ldots=$ $\hat{y}(k)=0, k \leq n$. When the output $\hat{y}(k)$ is predicted in time $k(k>n)$ by equations (11) and (13), the previous predicted values $\hat{y}(k-1), \hat{y}(k-2), \ldots \hat{y}(k-n)$ already exist. This processing method will cause the error between the predicted output and the measured data in the first few steps ineluctably, but after 20 to 40 iterative calculations, the errors between them will become smaller and smaller and gradually meet the precision requirement.

2.4. Model Accuracy Verification. In order to evaluate the model, the predicted output is computed by equations (7) and (13), which is also compared with the measured carriage-body acceleration.

We define the model residual $r_{e}$ as the difference between the predicted output $\hat{y}$ and the measured carriage-body acceleration $y ; r_{e}$ is computed as

$$
r_{e}=\widehat{y}-y .
$$

According to references $[16,18]$, if the model can accurately identify the relation between the input and the output, the distribution of the model residual obeys a normal distribution with a mean of zero, that is, $r_{e} \sim N\left(0, \sigma^{2}\right)$. The variance $\sigma^{2}$ is the mean deviation from the mean of the model residuals, reflecting the dispersion 
of the model residuals. The smaller $\sigma$ is, the smaller the model prediction residual deviates from the mean value, that is, the closer the measured data $y$ is to the predicted output $\hat{y}$. There are many ways to verify whether the series obey the normal distribution, such as the graphic method and statistics. In this study, the normal probability graph method is used to directly verify how the model residual $r_{e}$ normally distributes.

The similarity between the measured data $y$ and the predicted output $\hat{y}$ is expressed by the waveform comparisons and correlation coefficient. The correlation coefficient $\rho$ can measure the linear correlation between two sets of series. $\rho$ is defined as

$$
\rho=\frac{\sum_{i=1}^{M}\left(y_{i}-\bar{y}\right)\left(\hat{y}_{i}-\overline{\hat{y}}\right)}{\sqrt{\sum_{i=1}^{M}\left(y_{i}-\bar{y}\right)^{2}} \sqrt{\sum_{i=1}^{M}\left(\hat{y}_{i}-\overline{\bar{y}}\right)^{2}}},
$$

where $M$ is the number of the predicted data; $\bar{y}$ is the expectation of $y$, calculated by the formula $\bar{y}=\left(\sum_{i=1}^{M} y_{i} / M\right)$; and $\hat{y}$ is the expectation of $\hat{y}$, which is $\bar{y}=\left(\sum_{i=1}^{M} \hat{y}_{i} / M\right)$.

The absolute value of the correlation coefficient ranges from 0.0 to 1.0. When the absolute value of the correlation coefficient is greater than 0.8 , it is considered that there is a strong linear correlation between the two sets of series.

The measured data are independently divided into two parts, one is the training data which is used to train the model to get appropriate parameters, and the other is the test data which is used to verify the feasibility of the constructed model. The number of the training data is 80,000 , denoted as $N$ in equations (9) and (10). The relationships between several sets of data blocks mentioned above are shown in Figure 3, where different colors represent different datasets.

\section{Numerical Test}

According to the method in Section 2, models are constructed, respectively; the steps are described as follows: first, the input variables are identified by coherent analysis with the carriage-body acceleration in spatial domain; secondly, the model's parameters are determined by the measured data from the CRCIT; finally, the constructed model predicts the carriage-body acceleration, and the predicted output is also compared with the measured data.

3.1. Vertical Transfer Model. Figure 4 shows the power spectral density of track irregularity profile and the carriagebody vertical acceleration measured from the CRCIT on HSR line A.

In Figure 4, the PSD of the track irregularity profile locates mostly in the range $[0.008,0.018] \mathrm{m}^{-1}$ in the spatial frequency domain, corresponding to the wavelength range $[55,125] \mathrm{m}$, while the PSD of the carriage-body vertical acceleration locates mainly in the range $[0.02,0.04] \mathrm{m}^{-1}$ in the spatial frequency domain, corresponding with the wavelength range $[25,50] \mathrm{m}$. This means that not all track irregularities can cause the body of the vehicle to vibrate evidently in whole spatial frequency domain. To ensure

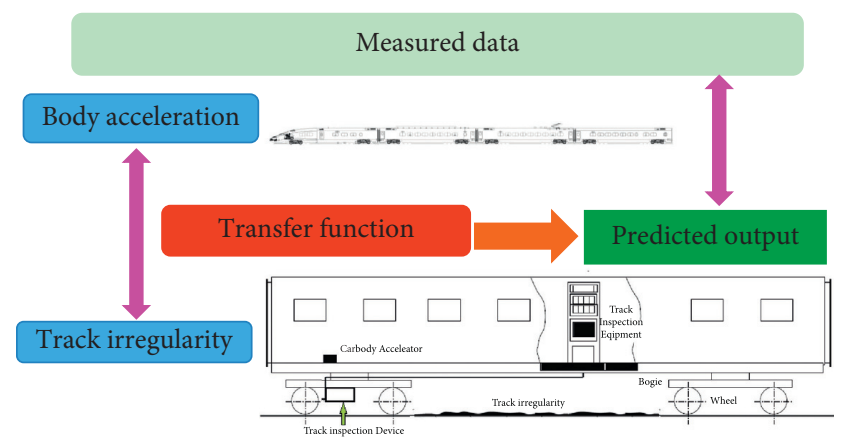

Figure 3: The relationship of the several sets of data.

comfortable and stable running, railway administration should give priority to track maintenance plan for repairing the track defects with unfavorable wavelength which can cause the vehicle to vibrate violently.

The maximum of the PSD of the carriage-body vertical acceleration in the spatial frequency is $0.0307 \mathrm{~m}^{-1}$, and its wavelength is $32.57 \mathrm{~m}$, where the PSD of track irregularity profile has local maximum as well. Those points periodically selected in Figure 4 show a series of harmonics with the fundamental wave frequency of $0.0307 \mathrm{~m}^{-1}$. This periodically local maximum indicates how the viaduct with $32.57 \mathrm{~m}$ approximately in the span length effect on the vertical vibration of the vehicle. With the increase in the harmonics order, the effect caused by the span length of the high-speed railway viaduct on the vibration of the vehicle attenuates quickly. Semilogarithmic graph can obviously display the changes of the harmonics with the order from one to eight in the PSD, as shown in Figure 5.

The right two local maxima in PSD in Figure 5(b) are caused by two factors: one is the track slab laid on the line where the CRCIT runs; the other is the out of round in the wheelset of the CRCIT.

Figure 6 shows the coherence function value between the track irregularity profile and the carriage-body vertical acceleration. The coherence function values in the range $[0.012,0.018] \mathrm{m}^{-1}$ and $[0.046,0.123] \mathrm{m}^{-1}$ are greater than 0.80 . In these sections, the ARX model can well identify the relation between the input and the output.

3.1.1. ARX Model Order Determination. After the input of the ARX model is selected, the reasonable model order is obtained by the AIC criteria calculated by equation (10), and the model parameters could be estimated by the measured data subsequently. The AIC values of the model with different orders are shown in Figure 7.

In Figure 7, the horizontal, longitudinal, vertical coordinate values of grid nodes are $n a$ (the order of the input), $n b$ (the order of the output), and the corresponding AIC values, respectively. In Figure 7, the AIC value has a point of inflection where the AIC value is the minimum. The numbers, $n a$ and $n b$, are, respectively, chosen according to the value of horizontal and longitudinal coordinates of the grid nodes. After the order of the model is determined, the polynomial 


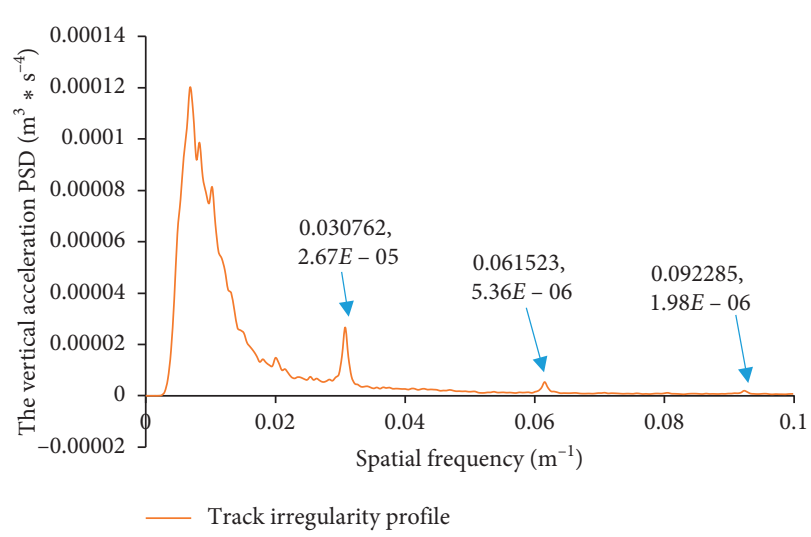

(a)

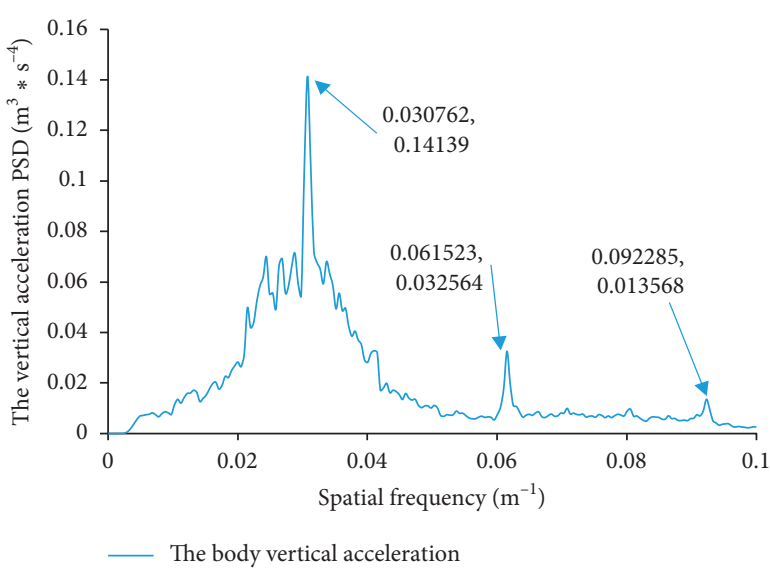

(b)

FIgURE 4: PSD of the measured data from line A.

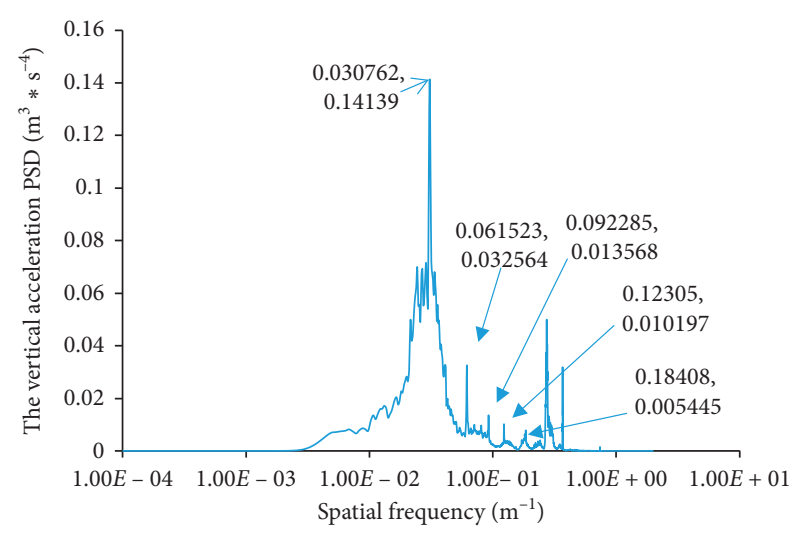

(a)

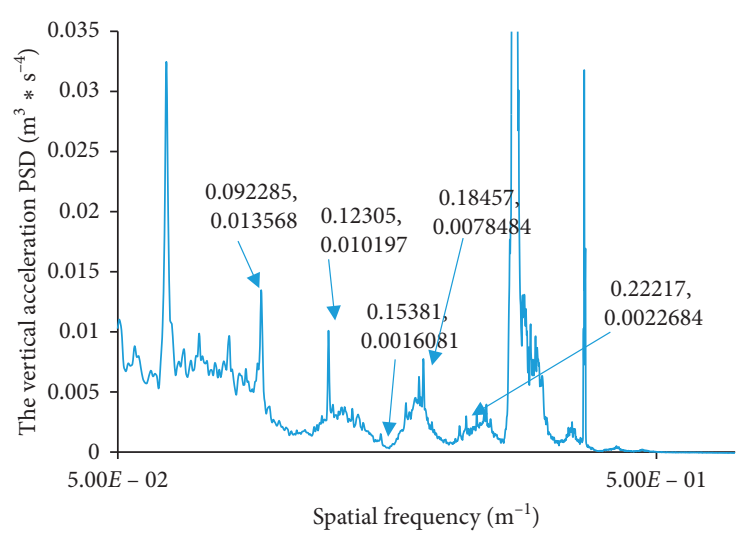

(b)

FIgURE 5: The PSD of the car-body vertical acceleration. (a) All band. (b) The details.

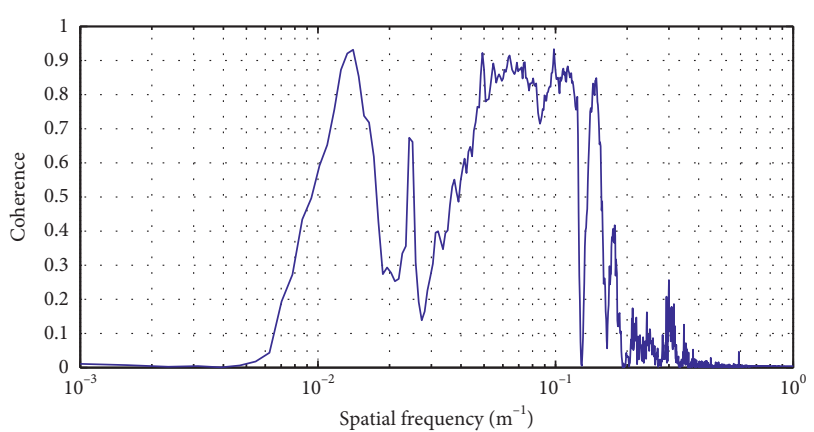

Figure 6: The coherence function value between the input and output.

coefficient, $\mathrm{A}(q)$ and $\mathrm{B}(q)$, in equation (3) can be estimated by the measured data subsequently.

The transfer function of the model is calculated by the equation (5), which reveals the transmitting characteristic from the track irregularity profile to the carriage-body vertical acceleration. Its amplitude-frequency characteristics and phase-frequency characteristics are shown in Figure 8 .

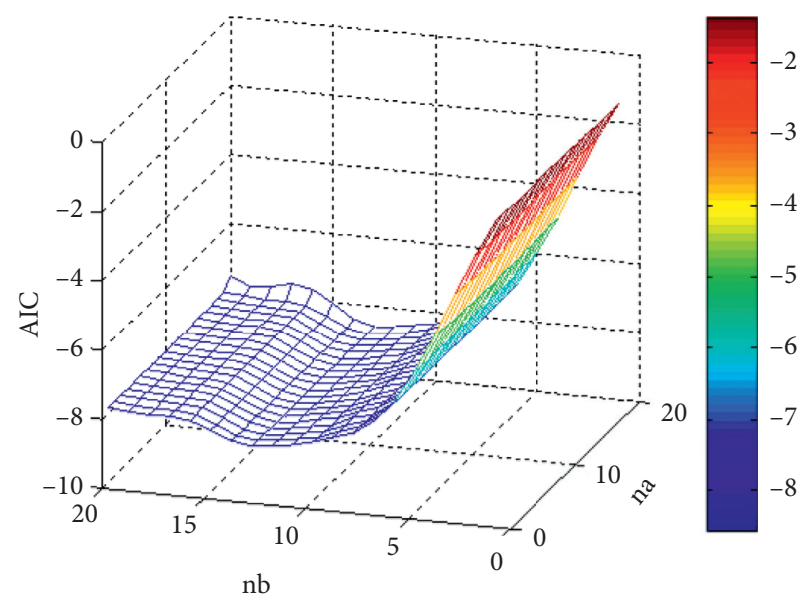

Figure 7: The relationship between AIC and the order of ARX Model.

3.1.2. Output Comparison of ARX Model. The carriage-body vertical acceleration is predicted by equation (8) and is compared with the measured carriage-body vertical 


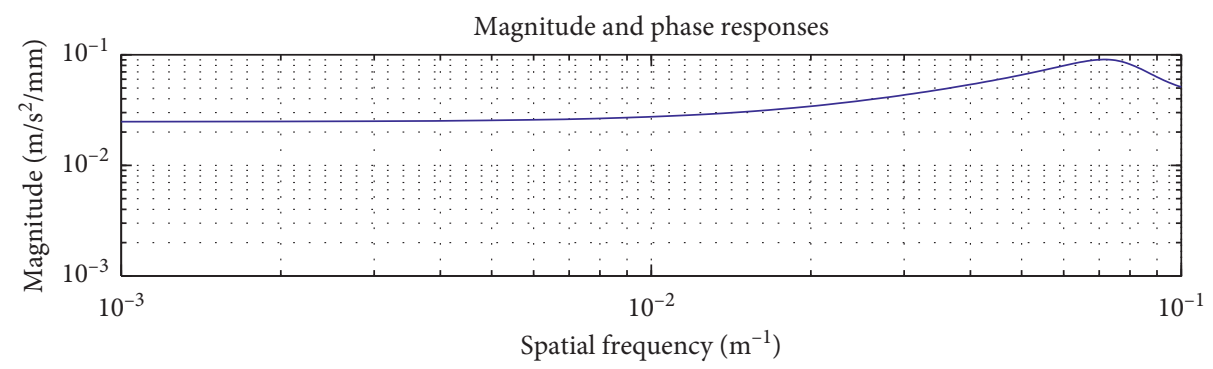

(a)

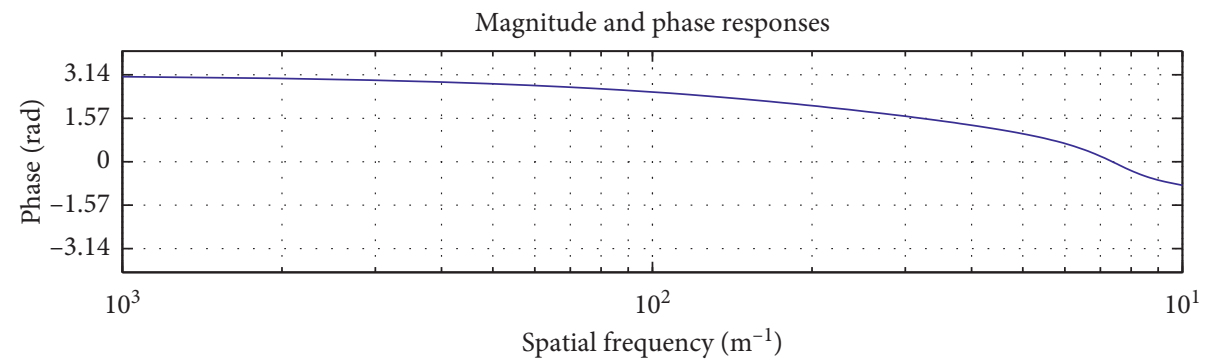

(b)

FIGURE 8: Transfer function amplitude and frequency characteristics of ARX model.

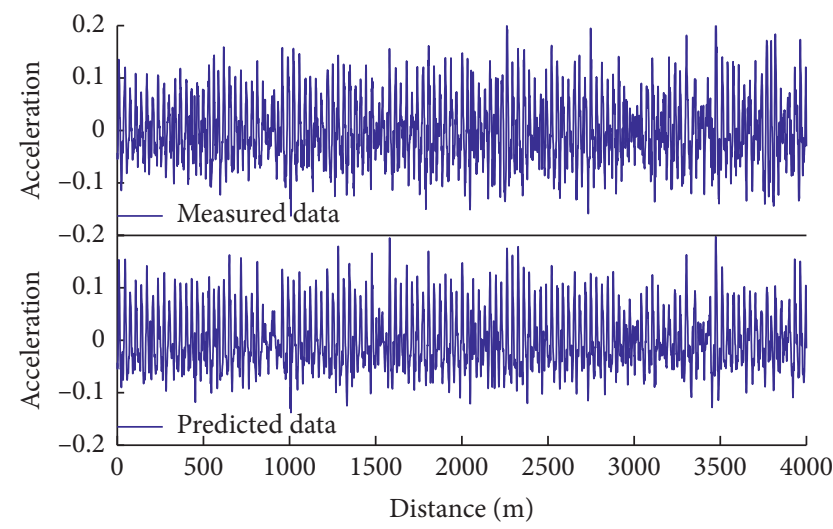

(a)

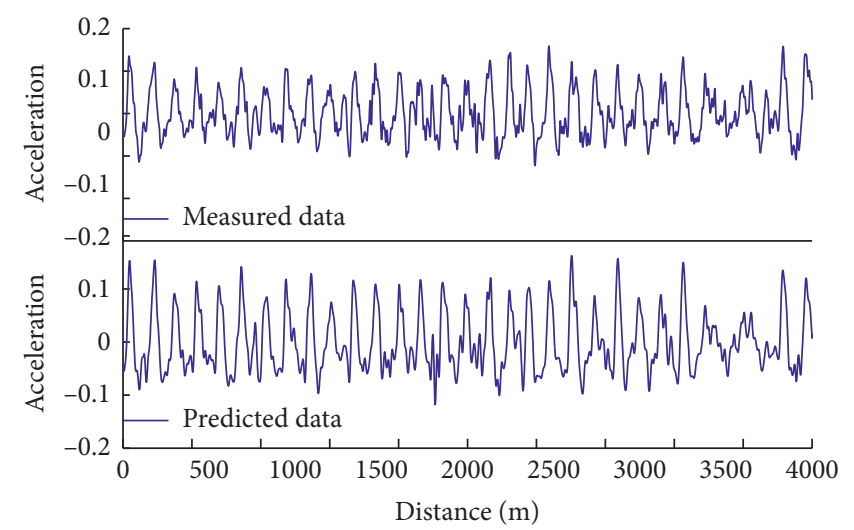

(b)

FIgURE 9: ARX model predicted data and measured data comparison of high-speed railway A. (a) The whole. (b) The details.

acceleration corresponding to the input. Two sets of data with a length of 16,000 sampling points are shown in Figure 9 , covering over $4000 \mathrm{~m}$ track lines. The correlation coefficient calculated by equation (15) between the predicted data and the measured data is 0.88 , so there is a strong linearity between the two sets of data.

In Figure 9, the predicted data show a good agreement with the measured data. The waveforms are similar and have the same changing trend. The ARX model residuals can be calculated by equation (14), and its distribution is shown in the normal probability diagram (Figure 10). By comparing the ARX model residuals and the standard normal distribution, the model residuals can be considered to obey normal distribution. The obedient parameters can be estimated by normal distribution function, that is, $r_{e} \sim N(-0.0013,0.0046)$, which approximately satisfies the zero-mean assumption and has small prediction error.
The measured data and the predicted data have similar waveforms not only in time domain but also in spatial frequency domain. Figure 11(a) shows the spectrum curve of them by means of spectrum analysis; the difference is very small in several frequency bands. Figure 11(b) shows the coherence function value between them, and there is a high coherence in the spatial frequency range $[0.01,0.05] \mathrm{m}^{-1}$, where the PSD of the carriage-body vertical acceleration locates mostly, as shown in Figure 5.

In summary, conclusion can be drawn that the constructed ARX model with appropriate parameters can well characterize the vertical transfer characteristics of the vehicle.

3.2. Lateral Transfer Model. Because of the complexity in the lateral transfer relation, the state space model constructed here is a type of multiple inputs and single output. Multiple 


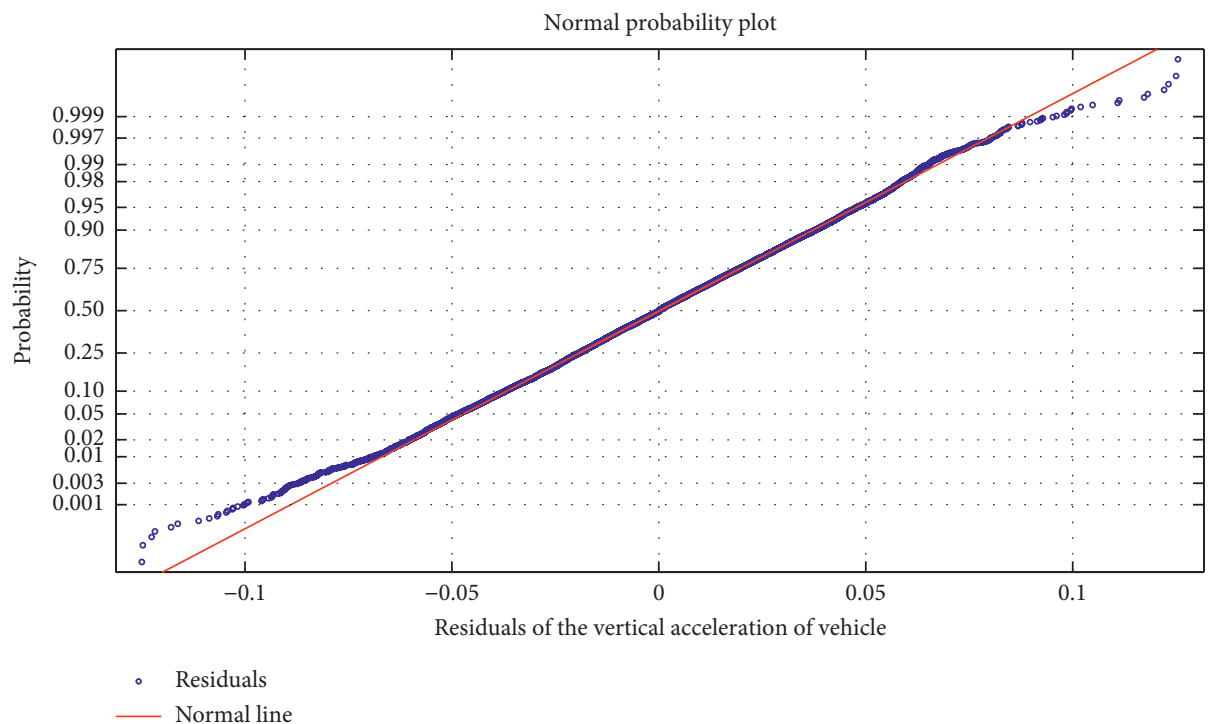

Figure 10: The distribution of the model residual.

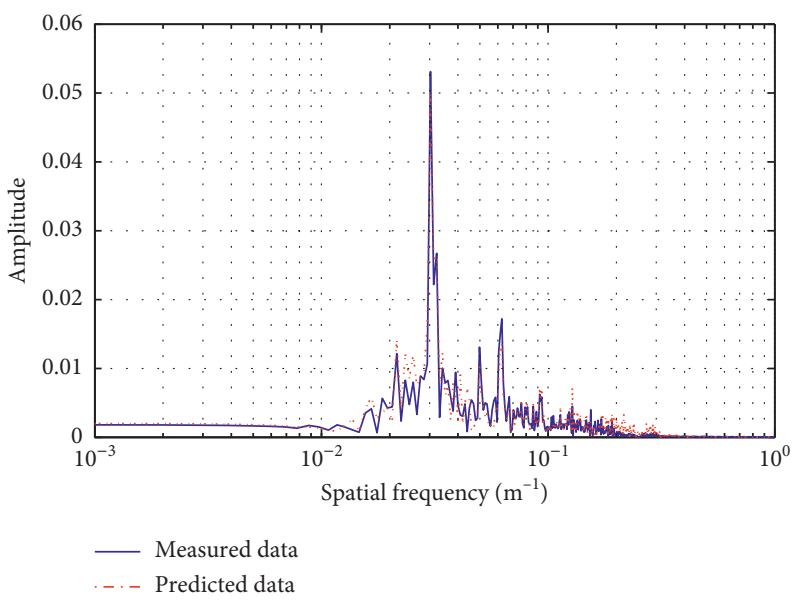

(a)

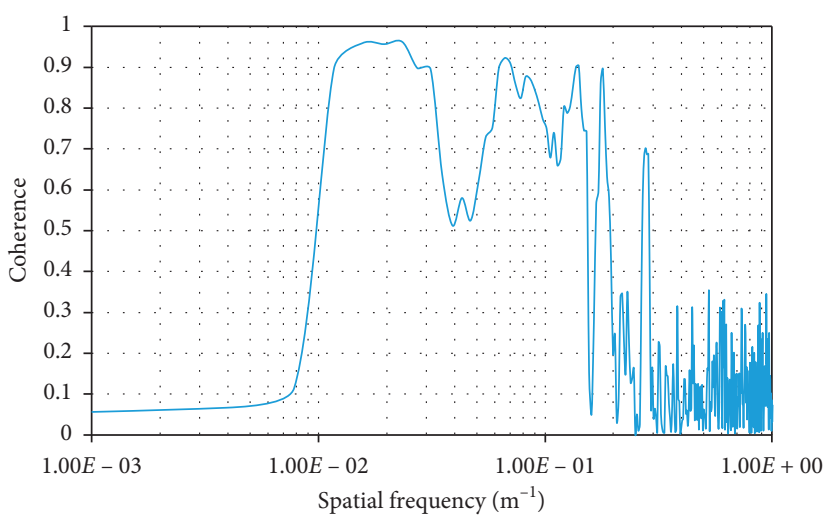

(b)

Figure 11: The relation of the predicted data and the measured data. (a) Spectrum curve. (b) Coherent curve.

inputs are selected variably according to the coherence function values in different spatial frequency bands. Black line in Figure 12 represents the PSD of the carriage-body lateral acceleration measured from high-speed railway line A; the coherent function curves between the carriage-body lateral acceleration and alignment curvature, cant, cross level, etc. The distribution of the carriage-body lateral acceleration mainly locates in the range $[0.025,0.045] \mathrm{m}^{-1}$. In the frequency band less than $0.009 \mathrm{~m}^{-1}$, the coherence function values between the carriage-body lateral acceleration and cant and curvature are greater than 0.80 . In the frequency band greater than $0.015 \mathrm{~m}^{-1}$, the coherence function values between the carriage-body lateral acceleration and alignment are larger.

In Figure 12, the coherence characteristics between the carriage-body lateral acceleration and different types of track irregularity vary in different spatial frequency bands. Hence, the sate space models are constructed correspondingly in different frequency sections; for values less than $0.009 \mathrm{~m}^{-1}$ and $[0.015$, $0.350 \mathrm{~m}^{-1}$, the model is named as the low-frequency model and mid-high-frequency models for the sake of distinction. In the low-frequency model, the inputs are curvature and cant; in the middle-high-frequency model, the input and output are obtained by a low-pass $0.009 \mathrm{~m}^{-1}$ filtering of the measured data; in the mid-high-frequency model, the inputs are alignment and cross level, which are obtained from the measured data adopted by a bandpass filtering of 0.015 to $0.350 \mathrm{~m}^{-1}$. The carriage-body lateral acceleration is filtered with the same filtering parameters accordingly.

3.2.1. Low-Frequency Model. Figure 13 shows that the AIC criteria values calculated by equation (10) vary with the orders of the model. The AIC criteria value is minimum when the order $n$ of the model equals 5 . Therefore, the model is constructed with the order $n=5$. 


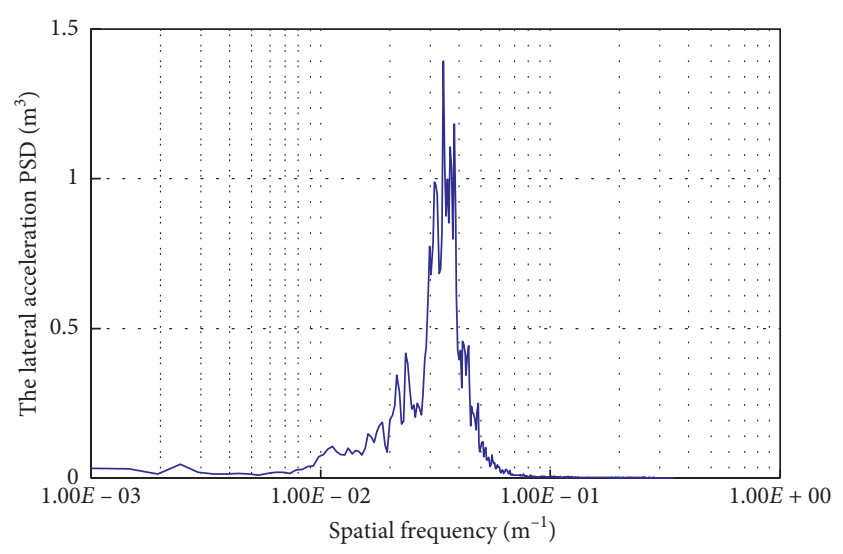

(a)

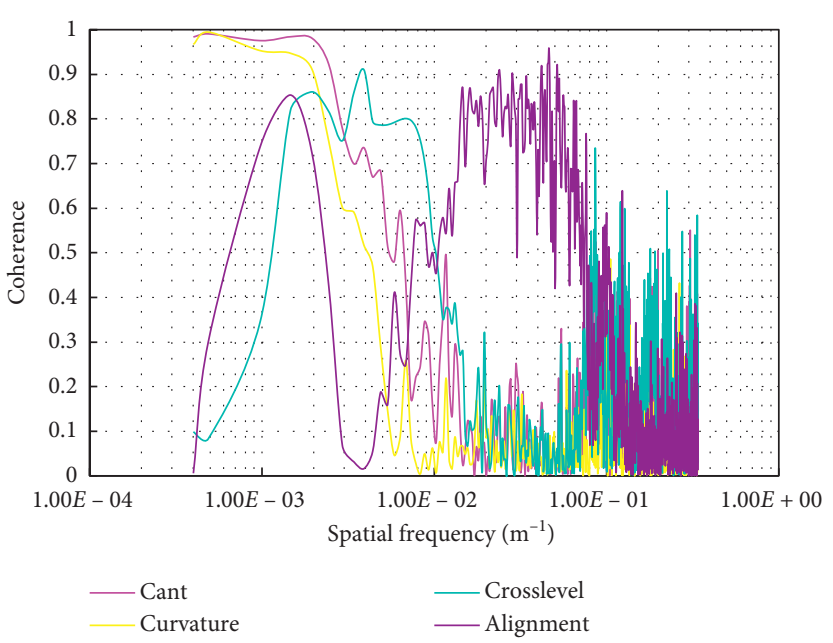

(b)

FIgURE 12: The car-body lateral acceleration PSD and coherence curves.

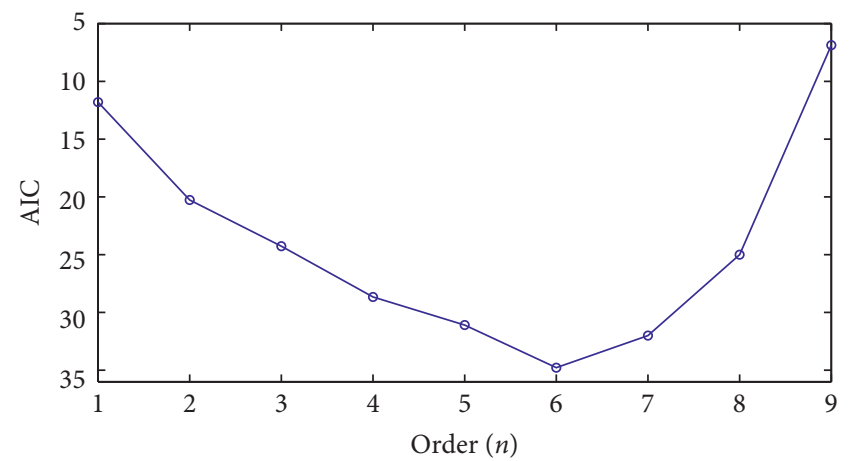

FIgUre 13: The relationship between AIC and order $n$ (low frequency).

The transfer function of the model can be calculated by equation (12) after the model parameter is determined, as shown in Figure 14. Compared with the transfer characteristics of curvature to the car body lateral acceleration, the change of the amplitude-frequency characteristics curve is gentle, and the phase-frequency characteristic curve shows that there is no phase difference between them.

The carriage-body lateral acceleration is predicted by equation (13) and compared with the measured carriagebody lateral acceleration, as shown in Figure 15(a). The correlation coefficient calculated by equation (15) between them is 0.98 ; there is a strong linearity between the two sets of data. Figure 15(b) shows a comparison between the two sets of data on the straight line in Figure 15(a).

In Figure 15, the predicted data have a good agreement with the measured carriage-body lateral acceleration. The model residuals defined by equation (14) are shown with cross sign scatter in the normal probability diagram; Figure 16 shows that the model residuals can be considered to obey normal distribution; the obedient parameters can be estimated by normal distribution function, $r_{e} \sim N(-0.0063,0.0232)$, which approximately satisfies the zero-mean assumption and has small prediction error.
3.2.2. Mid-High-Frequency Model. Figure 17 shows the AIC criteria values calculated by equation (10) varying with the orders of the model. The AIC criteria value is minimum when the order $n$ equals 6 . Therefore, the model is constructed when $n$ equals 6 .

The transfer function can be calculated by equation (12) after the model parameter is well determined. Figure 18 shows the amplitude and frequency characteristics of the transfer function between the inputs and output. The phasefrequency characteristic curves of both inputs to output show a sudden change from $-\pi$ to $\pi$, which means the delay in phase of the predicted data.

Figure 19(a) shows the predicted carriage-body lateral acceleration calculated by equation (13) and the measured data, covering over $16000 \mathrm{~m}$ track lines. The predicted output can be obtained according to equation (7), when the order of the model is $n$, the $n$ first predicted output values are set to 0 , and the error between the $(n+1)^{\text {th }}$ predicted value $\hat{y}(n+1)$ and $y(n+1)$ can probably be large, but after subsequently 20 to 40 iterative calculations, the error between the predicted output and the measured data will become smaller and smaller and the predicted output will gradually converge to the measured data.

The correlation coefficient calculated by equation (15) between the predicted data and the measured data is 0.85 . There is a strong linearity between the two sets of data. Figure 19(b) shows a comparison between the two sets of data on the straight line of the railway.

The predicted output generally coincides well with the measured carriage-body lateral acceleration. The model residuals defined by equation (14) are shown with cross sign scatter in the normal probability diagram in Figure 20. The model residuals can be considered to obey normal distribution; the obedient parameters can be estimated by normal distribution function, that is, $r_{e} \sim N(0.0003,0.0063)$, which satisfies the zero-mean assumption, and the variance of 0.0063 reflects the deviation from the residuals to the zero mean value; small variance 


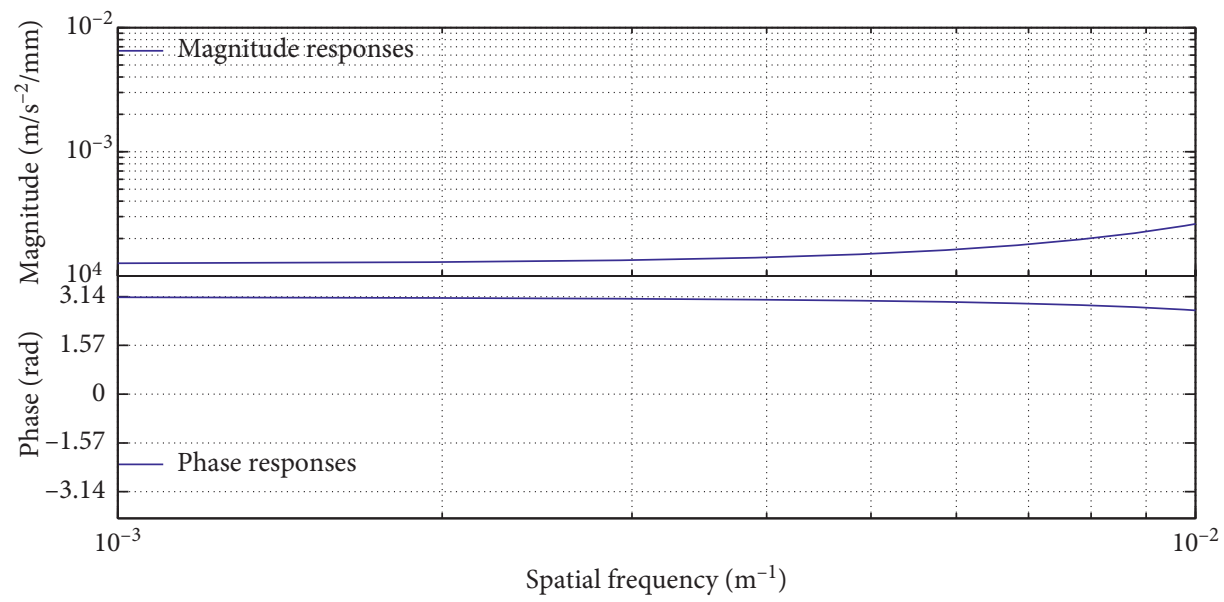

(a)

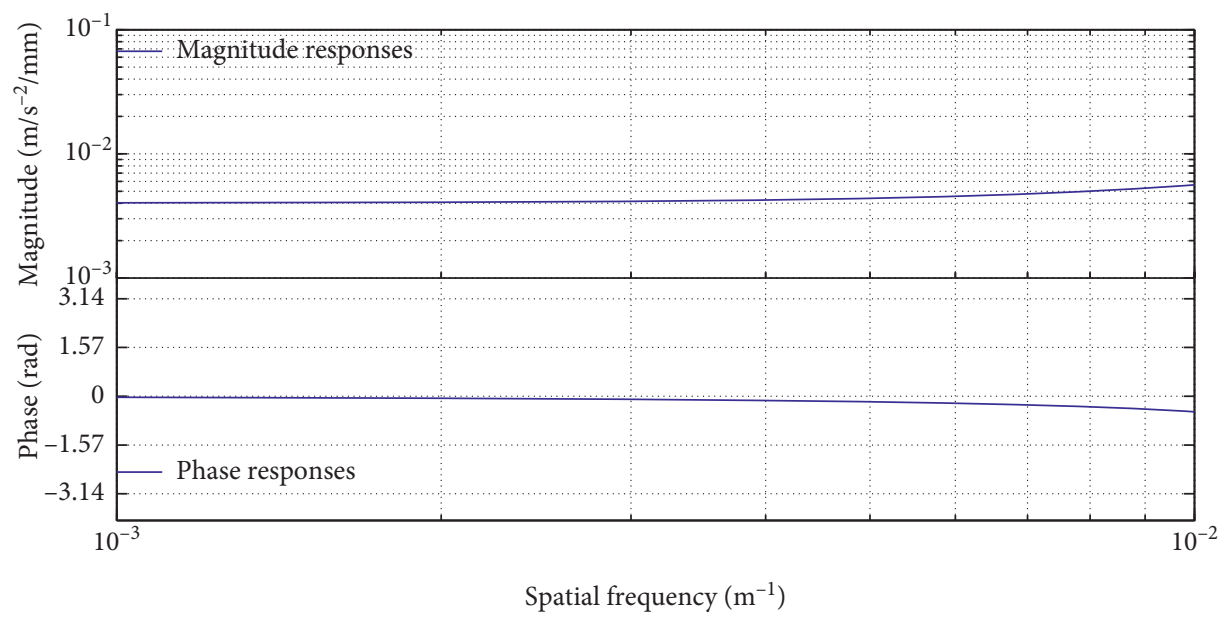

(b)

Figure 14: The transfer function characteristics curve (low-frequency model). (a) Curvature to the lateral acceleration. (b) Cant to the lateral acceleration.

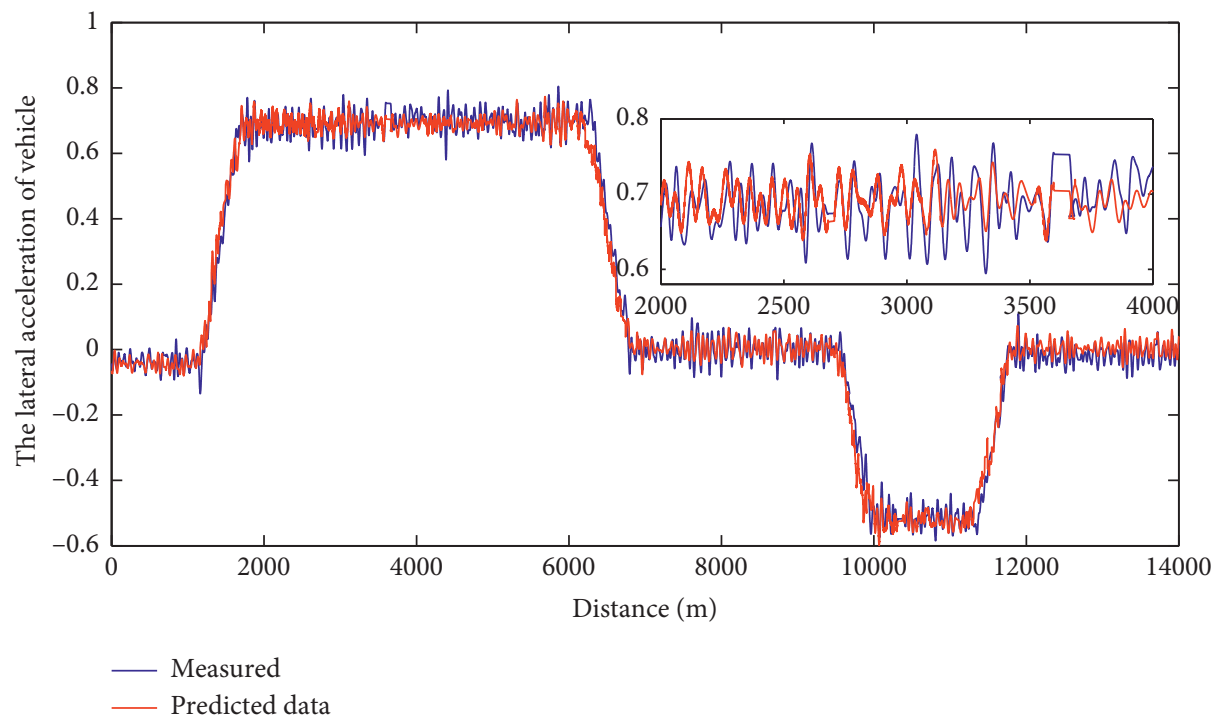

(a)

Figure 15: Continued. 


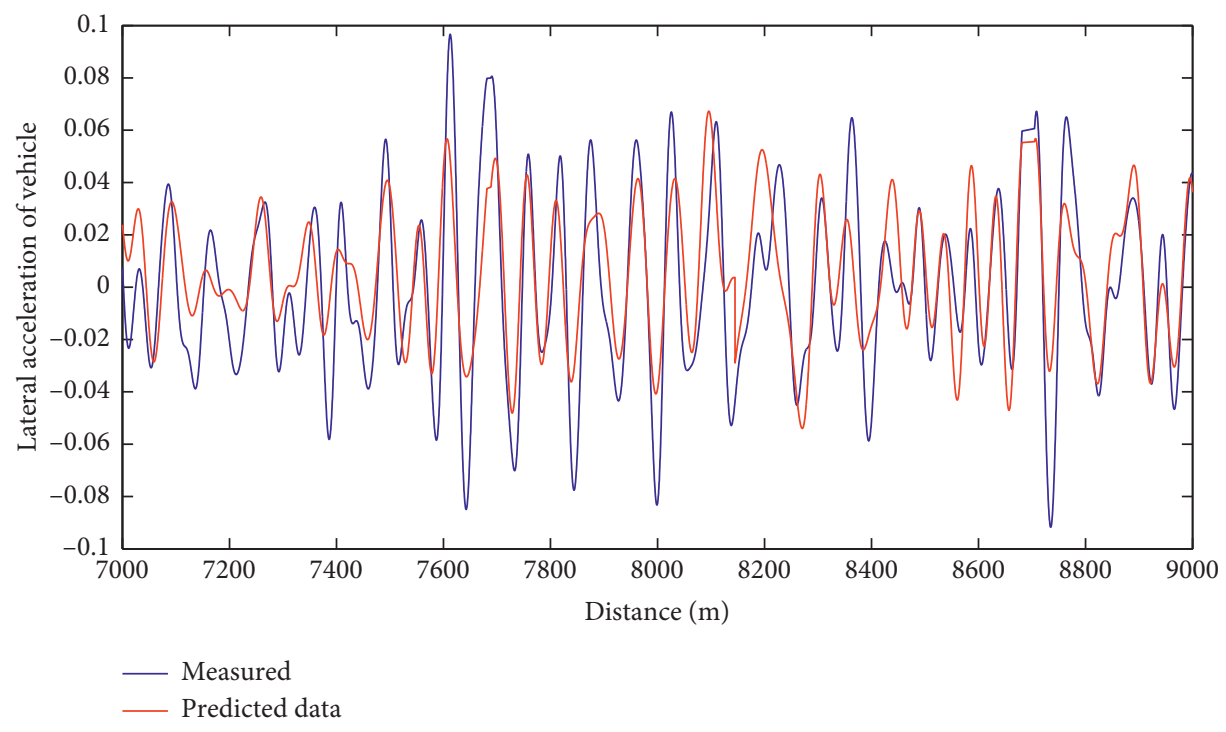

(b)

Figure 15: The comparison of measured data and predicted data (low frequency). (a) The whole. (b) Straight line.

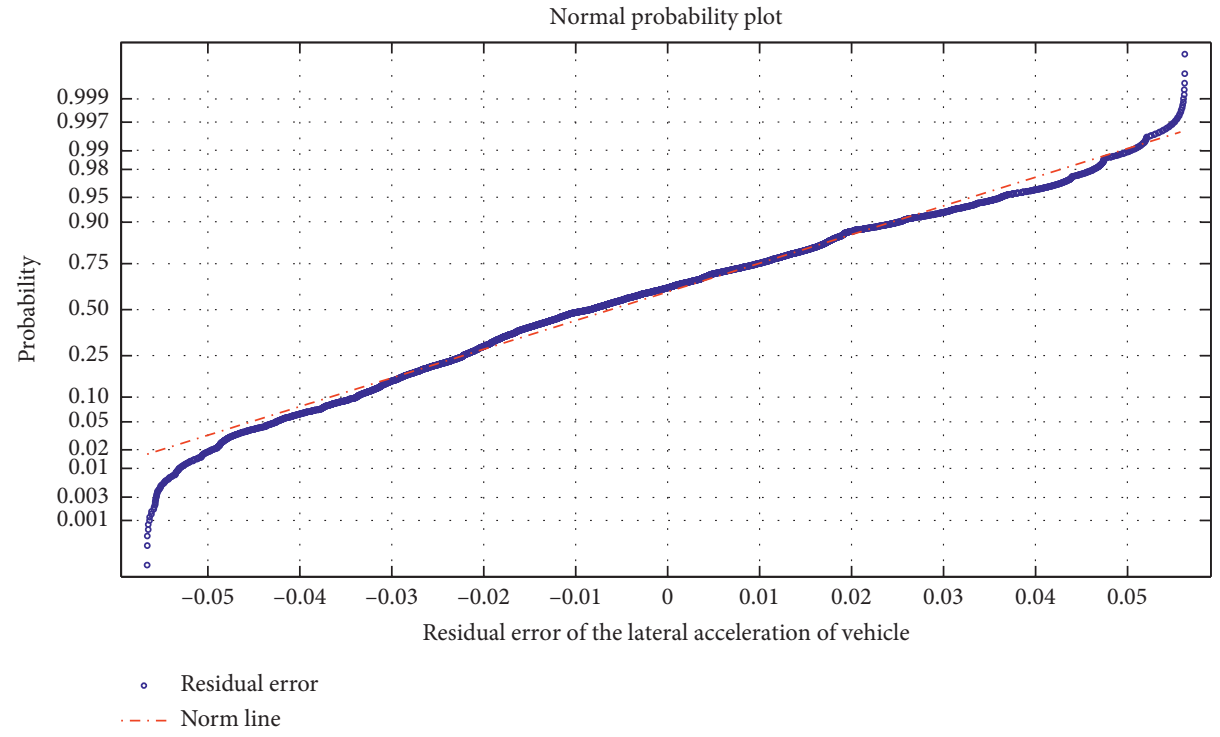

FIgURE 16: Model residual distribution of the low-frequency state space model.

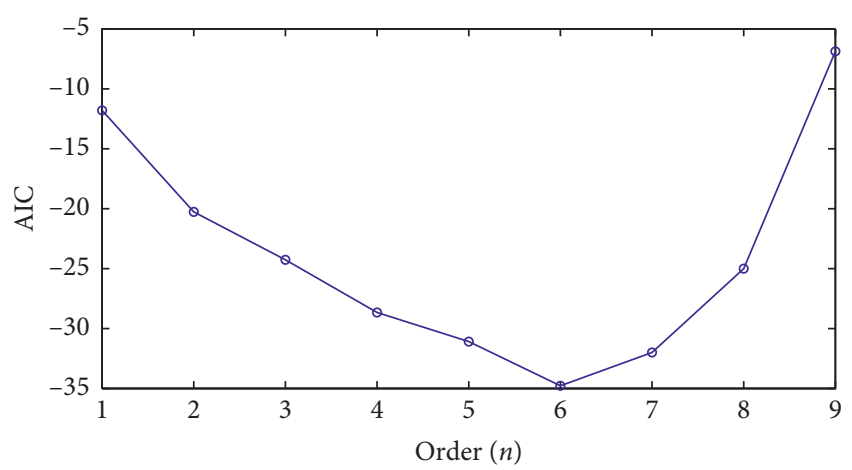

FIGURE 17: Relation between AIC and the $n^{\text {th }}$ order of the model. means that the model output value is very close to the measured value.

The correlation coefficient between the two data sets is higher than 0.8 , which means there are strong linear correlations between them. This also affirms that the transfer function is correct, and the transfer function can identify accurately the relationship between the track irregularity and the carriage-body lateral acceleration.

3.2.3. Model Output Comparison. This section shows the comparison between the state space model and the ARX model in identifying the lateral transfer characteristics of the vehicle. Figure 21 shows the predicted output by the state 


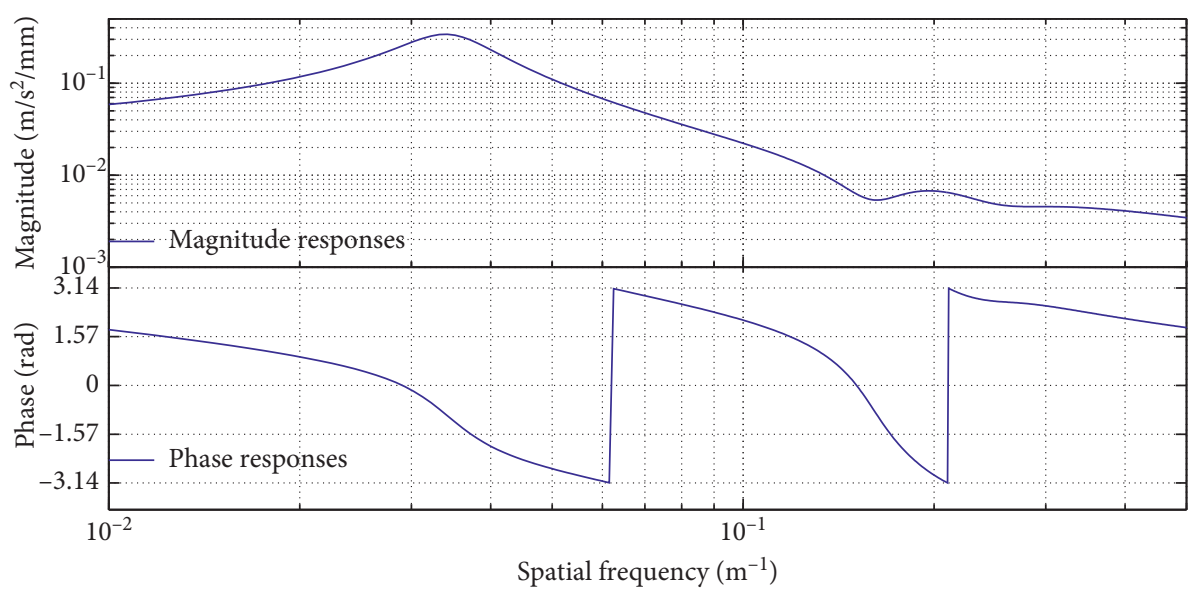

(a)

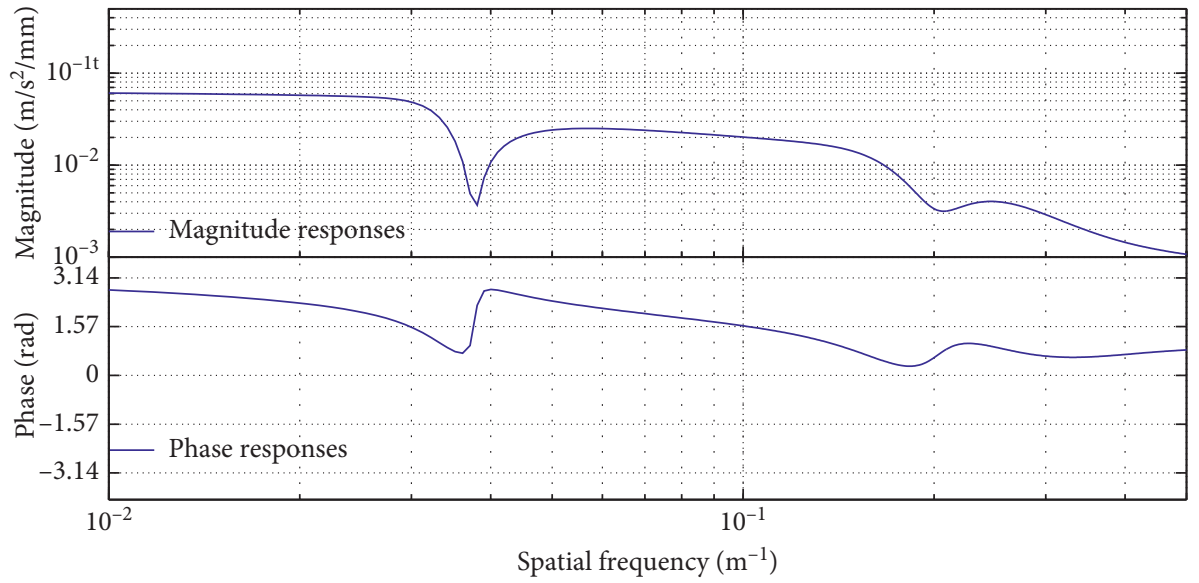

(b)

Figure 18: State space method transfer function characteristics ( $6^{\text {th }}$ high-frequency model). (a) Alignment to the lateral acceleration. (b) Cross level to the lateral acceleration.

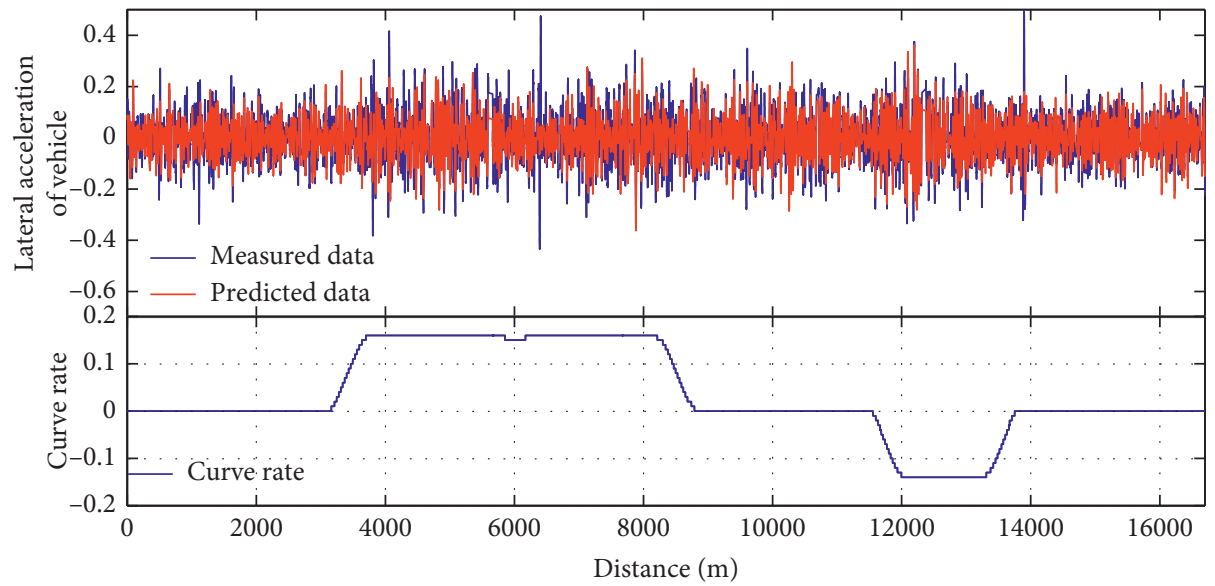

(a)

Figure 19: Continued. 


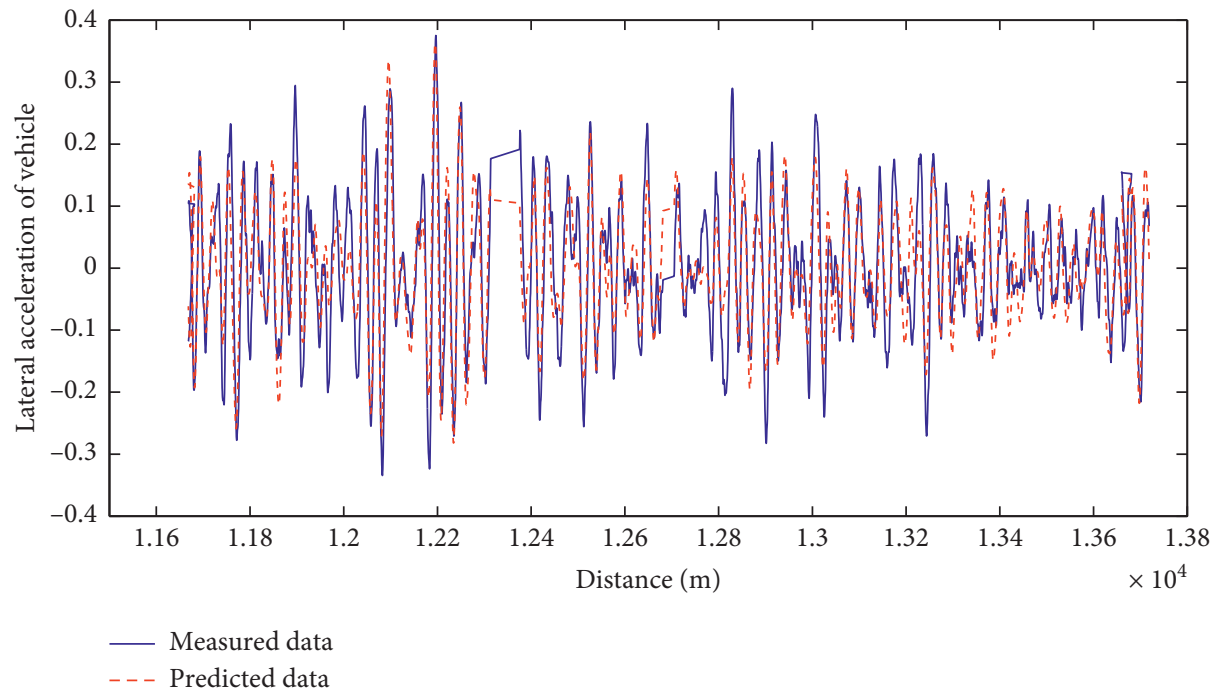

(b)

FIgURE 19: Comparison of measured data and predicted data by state space model (mid-high frequency). (a) The whole. (b) Straight line.

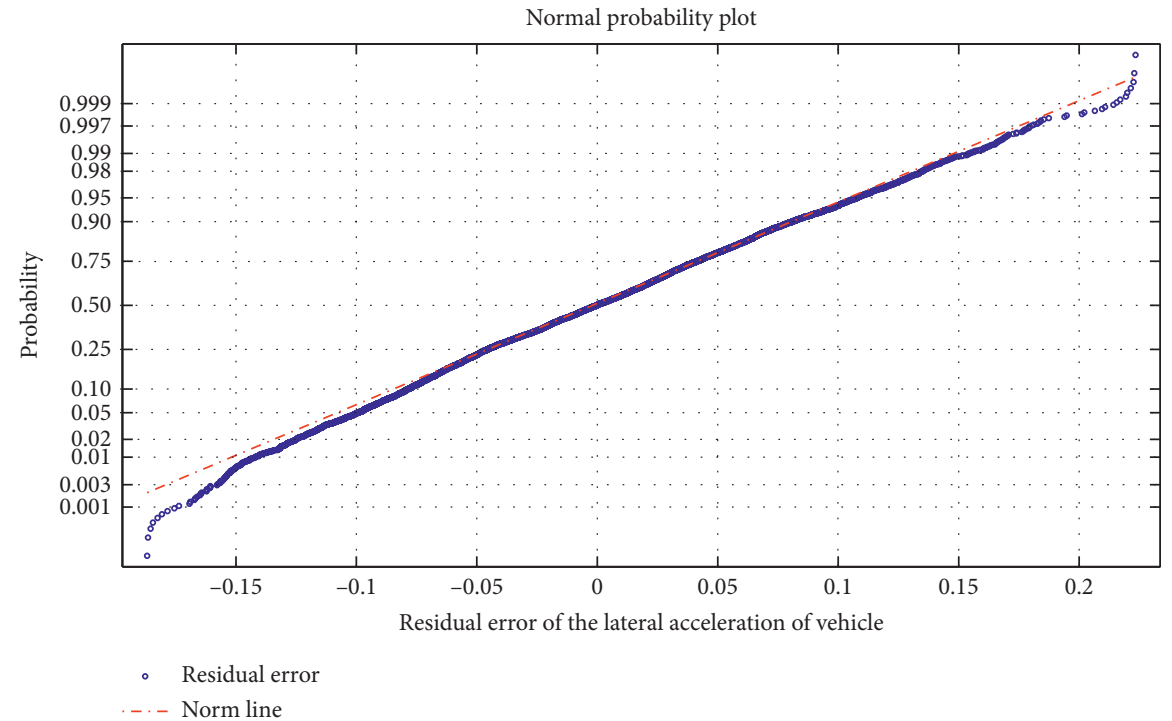

Figure 20: Distribution curves of model residuals.

苞

芯

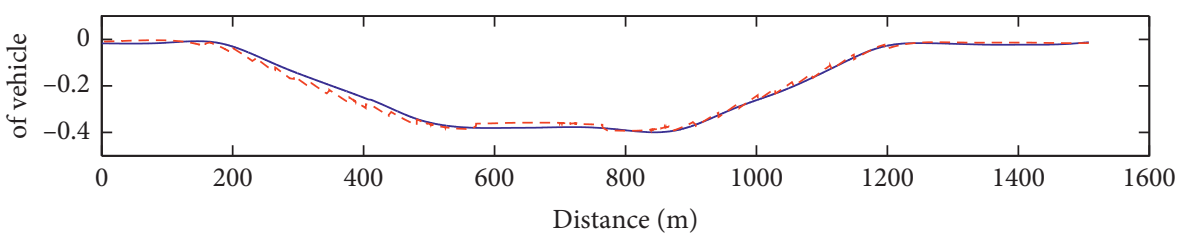

- Measured data

- - - Predicted data

(a)

FIGURE 21: Continued. 
苞

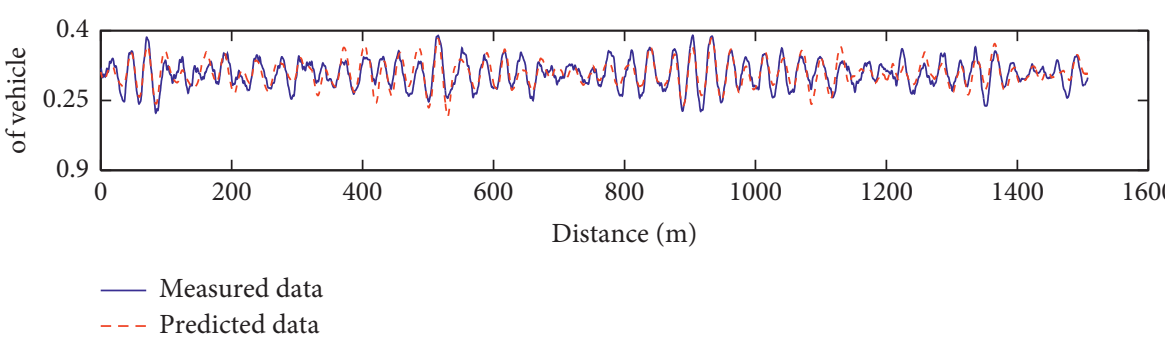

(b)

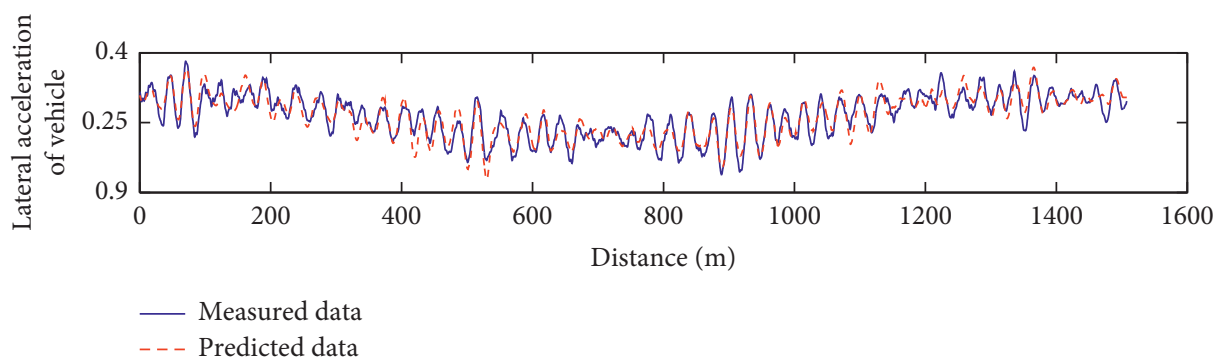

(c)

FIGURE 21: Comparison between predicted output and measured data (state space model).

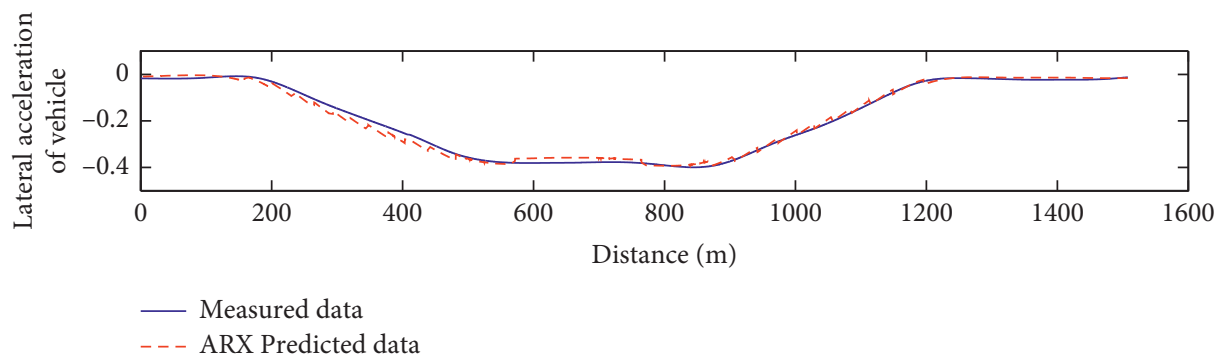

(a)

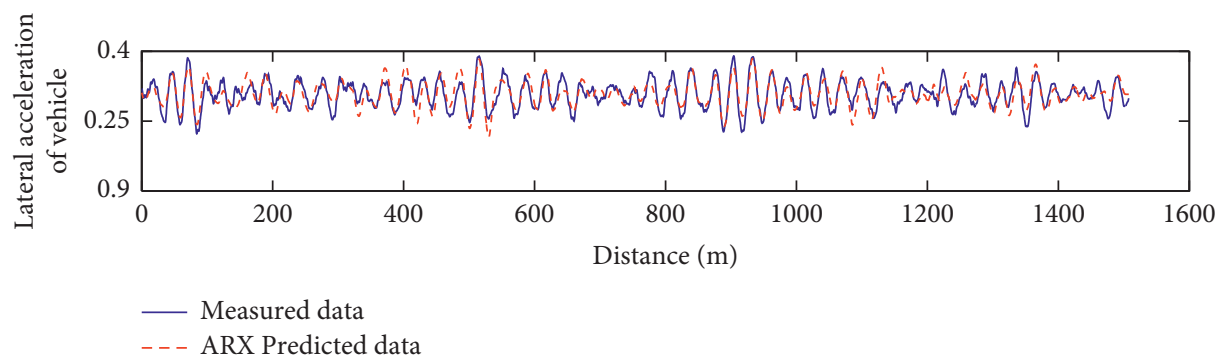

(b)

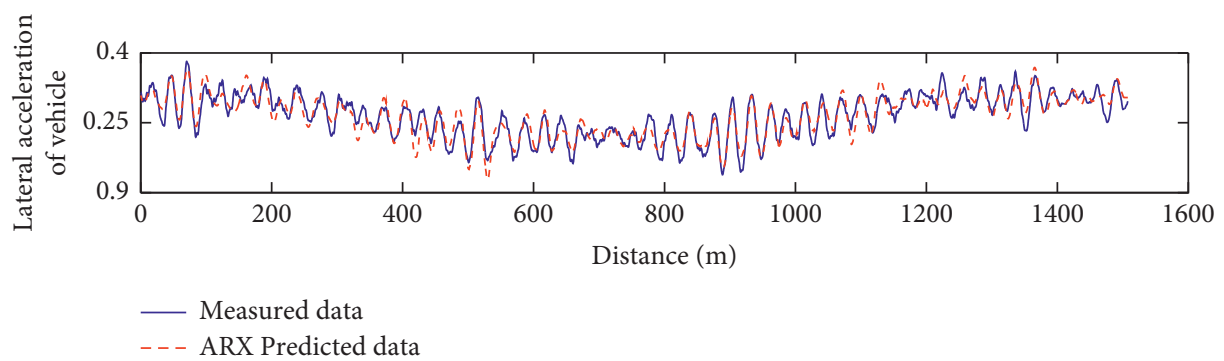

(c)

FIGURE 22: Comparison between predicted output and measured data (ARX model). 
space model and the measured data. The selected section is 1500 meters in length with a radius of 12,000 meters and two straight lines. The total number of the predicted output is 6000 . Figure 22 illustrates the results obtained by the ARX model.

From the above figures, although the predicted output in a straight line and a curve by the state space and ARX models is alike in waveform, the correlation coefficients of the two sets of data in Figure 21 in low frequency, mid-high frequency, and the whole frequency are 0.97, 0.87, and 0.94, respectively. The correlation coefficients in Figure 22 are $0.85,0.78$, and 0.81 , respectively. The state space model can more suitably identify the transfer relation between the track irregularity and the carriage-body lateral acceleration. The accuracy of the ARX model identification has to be improved.

\section{Conclusions}

The effect on the vehicle operation from the wavelength of the track irregularity is more complex than that from the amplitude of track irregularity. The ways to eliminate the two types of track roughness are also different. Studying the transfer relation between track irregularity and the carriagebody acceleration helps obtain the track roughness with unfavorable wavelength. The relation model is built via coherent analysis and system identification method, and the feasibility of the model is discussed by comparing the predicted data with the measured data from the CRCIT. The main conclusions are summarized as follows.

A strong correlation exists between the track irregularity and the carriage-body vertical acceleration in spatial frequency range $[0.012,0.018] \mathrm{m}^{-1}$ and $[0.046,0.123] \mathrm{m}^{-1}$. The ARX model is built based on the frequency bands and the model parameters determined through AIC rule. The transfer function waveform is obtained from the model. By comparing the predicted output and the measured data, the results show that the ARX model can identify the vertical transmission characteristics from track irregularity profile to the carriage-body vertical acceleration.

Based on the coherence analysis of the track irregularity and the carriage-body lateral acceleration, the state space models are constructed in two spatial frequency bands less than $0.009 \mathrm{~m}^{-1}$ and $[0.015,0.350] \mathrm{m}^{-1}$, and the reasonable order and parameters are obtained by training the model with the measured data. The model residuals approximately conform to the assumption of zero normal distribution. The state space model with proper order can identify the lateral transmission characteristics from alignment, cant, cross level, to the carriage-body lateral acceleration.

The distributions of the track irregularity and the carriage-body acceleration do not overlap completely; it is a wise choice to eliminate the targeted rail defects with unfavorable wavelength. By using the relation model and the transfer characteristic, the influence from the track irregularity with different wavelengths on the carriage-body of the vehicle can be analyzed; railway administration should give priority to track maintenance plan for repairing the rail defects with unfavorable wavelength where the track irregularity can cause the vehicle to vibrate violently.
The transfer characteristics vary with the vehicle operating speed and the vehicle suspension, and the parameters of the model constructed by the ARX model or the state space model also vary with the vehicle operation speed and the type of the vehicle. The change rule of the models with different speeds and the suspensions will be emphasized based on the diversified high-speed operation in China in the further investigation.

\section{Data Availability}

The data used to support the findings of this study are available from the corresponding author upon request.

\section{Conflicts of Interest}

The authors declare that they have no conflicts of interest.

\section{Acknowledgments}

This study was financially supported by the Science and Technology Research and Development Project of China Railway Corporation (2017G011-E and 2018YJ042).

\section{References}

[1] H. Xia, N. Zhang, and W. Guo, "Self-excitations of trainbridge coupling vibration system," Dynamic Interaction of Train-Bridge Systems in High-Speed Railways, Springer, Berlin, Germany, pp. 149-190, 2018.

[2] B. Luber, "Railway track quality assessment method based on vehicle system identification," $e$ \& i Elektrotechnik und Informationstechnik, vol. 126, no. 5, pp. 180-185, 2009.

[3] X. Hu, M. Hou, C. Chang, Li Lan, and Bo Chen, "Relationship with vehicle response based on the stationary-state and nonstationary-state vibration signal analysis," China Railway Science, vol. 33, no. 8, pp. 46-51, 2012.

[4] X. Kang, W. Wang, and J. Liu, "Research on comprehensive evaluation system for track irregularity of high-speed railway based on RAMS," China Railway Science, vol. 34, no. 2, pp. 13-17, 2013.

[5] M. Kargarnovin, D. Younesian, D. Thompson, and C. Jones, "Ride comfort of high-speed trains travelling over railway bridges," Vehicle System Dynamics, vol. 43, no. 3, pp. 173-197, 2005.

[6] M. Odashima, S. Azami, Y. Naganuma, H. Mori, and H. Tsunashima, "Track geometry estimation of a conventional railway from car-body acceleration measurement," Mechanical Engineering Journal, vol. 4, no. 1, pp. 16-00498, 2017.

[7] K. Wang, W. Zhai, J. Liu, Q. Feng, C. Cai et al., "Effect of rail irregularity wavelength on lateral running comfort of speedraised train," Journal of Traffic \& Transportation Engineering, vol. 7, no. 1, pp. 1-5, 2007.

[8] J. Xu, P. Wang, Li Wang, and J. Xiao, "Research on the distribution characteristics and influence factors of sensitive wavelength of track vertical profile irregularity," Journal of the China Railway Society, vol. 37, no. 7, pp. 72-78, 2015.

[9] K. Youcef, T. Sabiha, D. El Mostafa, D. Ali, and M. Bachir, "Dynamic analysis of train-bridge system and riding comfort of trains with rail irregularities," Journal of Mechanical Science and Technology, vol. 27, no. 4, pp. 951-962, 2013. 
[10] L. Niu, "Correlation model of track irregularity and car-body vibration based on BP neural network," China Railway Science, vol. 37, no. 2, pp. 26-32, 2016.

[11] P. Czop, K. Mendrok, and T. Uhl, "Application of inverse linear parametric models in the identification of rail track irregularities," Archive of Applied Mechanics, vol. 81, no. 11, pp. 1541-1554, 2011.

[12] C. H. Lee, C. W. Kim, T. M. Kawatani, N. Nishimura, and T. Kamizono, "Dynamic response analysis of monorail bridges under moving trains and riding comfort of trains," Engineering Structures, vol. 27, no. 14, pp. 1999-2013, 2005.

[13] S. Javad, R. Siamak, and K. Amin, "Effect of rail irregularities on ride comfort of train moving over ballast-less tracks," International Journal of Structural Stability and Dynamics, vol. 19, no. 6, Article ID 1950060, 2019.

[14] Ji Wang and X. Hu, Application of MATLAB in Vibration Signal Processing, China Water Conservancy and Hydropower Publishing House, Beijing, China, 2006.

[15] A. Furukawa and A. Yoshimura, "A method to predict track geometry-induced vertical vehicle motion," Quarterly Report of RTRI, vol. 45, no. 3, pp. 142-148, 2004.

[16] V. Balakrishnan, L. Ljung, System Identification: The Theory for the User, Prentice-Hall, Englewood Cliffs, NJ, USA, 2nd edition, 1999.

[17] R. Shanmugam, P. J. Brockwell, and R. A. Davis, "Introduction to time series and forecasting," Technometrics, vol. 39, no. 4, p. 426, 1997.

[18] J. D. Cryer Kungsik Chan, Time Series Analysis with Applications in $R$, Springer Science and Business Media Limited Liability Company, New York, NY, USA, 2nd edition, 2008. 